\title{
Vorwort des Herausgebers
}

Die Niederschlagung des Kapp-Lüttwitz-Putsches durch die einig handelnde Arbeiterklasse ist ein zentrales Ereignis der Geschichte der deutschen Arbeiterbewegung und des deutschen Volkes, dem die besondere Aufmerksamkeit der marxistisch-leninistischen Geschichtswissenschaft gilt. Damals bewiesen die Arbeiter, daß die Aktionseinheit - eine unabdingbare Voraussetzung für den siegreichen Kampf der Arbeiterklasse in der Epoche des Ubergangs vom Kapitalismus zum Sozialismus - in der Praxis des Klassenkampfes mit Erfolg verwirklicht werden kann. Die Herausbildung der Aktionseinheit der verschiedenen Abteilungen der Arbeiterbewegung im antiimperialistischen Kampf ist nicht nur eine Frage des historischen Interesses, sondern ist darüber hinaus von höchst aktueller politischer Bedeutung.

So zog die Internationale Beratung der kommunistischen und Arbeiterparteien im Juni 1969 in Moskau aus der wissenschaftlichen Analyse des. internationalen Klassenkampfes die Schlußfolgerung, daß der Kampf gegen den Imperialismus auf eine neue Stufe gehoben werden kann. In der gegenwärtigen Etappe des Klassenkampfes hat sich angesichts des Vormarsches der drei revolutionären Hauptströmungen unserer Zeit - des sozialistischen Weltsystems, der Arbeiterbewegung in den kapitalistischen Ländern und der nationalrevolutionären Befreiungsbewegung - der Widerspruch zwischen den beiden entgegengesetzten gesellschaftlichen Systemen, der Hauptwiderspruch unserer Epoche, bedeutend vertieft. Neue revolutionäre Möglichkeiten entstehen, um der imperialistischen Politik der Aggression und des Krieges endgültig eine Niederlage zu bereiten. Perspektiven und Ergebnisse des antiimperialistischen Kampfes hängen jedoch in erster Linie davon ab, wie es gelingt, unter der Führung der kommunistischen Weltbewegung das Bündnis aller antiimperialistischen Kräfte zu entwickeln und zu festigen. „Im Bewußtsein ihrer historischen Verantwortung“, heißt es deshalb im Hauptdokument, ,wenden sich die auf der Beratung vertretenen kommunistischen und Arbeiterparteien an alle Kommunisten der Welt, an alle Gegner des Imperialismus, an alle, die zum Kampf für 
Frieden, Freiheit und Fortschritt bereit sind, mit dem Aufruf zur Aktionseinheit. “1

Beim Rückblick auf den Kapp-Putsch werden in der Aktionsgemeinschaft der Arbeiterklasse und der Volksmassen gegen die Putschisten die Potenzen der demokratischen, antiimperialistischen und antimilitaristischen Kräfte im Kampf gegen Imperialismus und Militarismus deutlich. Die hier in erbittertem Ringen mit dem Klassengegner gesammelten Erfahrungen vermitteln sowohl in nationaler als auch in internationaler Hinsicht wichtige Lehren und Schlußfolgerungen für die gegenwärtige Auseinandersetzung zwischen den Systemen des Sozialismus und des Imperialismus.

Die Epoche des Ubergangs vom Kapitalismus zum Sozialismus wurde durch den Sieg der Großen Sozialistischen Oktoberrevolution eingeleitet, und die Auseinandersetzungen zwischen den beiden gesellschaftlich entgegengesetzten Systemen bildeten von nun an den Hauptinhalt der Weltgeschichte.

Die Oktoberrevolution übte auf die Klassenkämpfe in Deutschland, das zum Knotenpunkt der imperialistischen Widersprüche geworden war ${ }^{2}$, einen tiefen Einfluß aus. Die gesellschaftlichen Widersprüche, die in Rußland durch die erste siegreiche proletarische Revolution bereits überwunden worden waren, drängten besonders auch in Deutschland seit der Jahrhundertwende nach einer Lösung. Erstmalig in der Novemberrevolution versuchten die deutschen Arbeiter und die anderen Werktätigen, die Macht des deutschen Imperialismus und Militarismus zu brechen und den Ubergang vom Kapitalismus zum Sozialismus zu vollziehen. ${ }^{3}$

Die neue weltgeschichtliche Situation veranlaßte den deutschen Imperialismus, seine Strategie und Taktik den veränderten Bedingungen anzupassen. Angesichts der tiefgreifenden Auseinandersetzung in Deutschland sahen führende Vertreter des Monopolkapitals und des Militarismus in der Ausnutzung und Förderung des bürgerlichen Einflusses in der

1 Internationale Beratung der kommunistischen und Arbeiterparteien Moskau 1969, Berlin 1969, S. 36.

2 Geschichte der deutschen Arbeiterbewegung in acht Bänden, hg. v. Institut für Marxismus-Leninismus beim ZK der SED, Berlin 1966, Bd. 3, S. 228.

3 Vgl. hierzu Diehl, Ernst, Die Bedeutung der Novemberrevolution 1918, in: ZfG, 1-2/1969. 
Arbeiterbewegung die entscheidende Voraussetzung, um ihre Klassenherrschaft auch weiterhin zu behaupten. Mit Hilfe der rechten SPDund Gewerkschaftsführer sollte die Krise des imperialistischen Herrschaftssystems überwunden werden, wobei der enge und untrennbare Zusammenhang zwischen Imperialismus und Opportunismus eine neue Entwicklungsstufe erreichte. Die Zusammenarbeit zwischen Imperialisten und Opportunisten vertiefte die Spaltung der Arbeiterklasse und erschwerte die Durchführung einheitlicher Aktionen gegen das Monopolkapital. Um die werktätigen Massen an den imperialistischen Staat zu binden, sahen sich die deutschen Monopolherren gezwungen, die Formen der Machtausübung zu verändern und auf sozialpolitischem Gebiet Zugeständnisse zu machen. Während sie einerseits bürgerlich-demokratische Illusionen in der Arbeiterklasse ausnutzten, entfachten sie andererseits gegen die revolutionäre Vorhut der deutschen Arbeiterklasse eine infame Hetze, die von brutalem Terror begleitet wurde. Die ideologische Hauptwaffe der deutschen Bourgeoisie und der mit ihr verbundenen rechten sozialdemokratischen Führer war der Antikommunismus; er richtete sich sowohl gegen die revolutionäre deutsche Arbeiterbewegung als auch gegen Sowjetrußland.

Vom Ausgang des Klassenkampfes in Deutschland, das unter den Auswirkungen der Großen Sozialistischen Oktoberrevolution und der Kriegsniederlage zum schwächsten Kettenglied geworden war, hing aber zumindest das Schicksal des Imperialismus in Europa weitgehend ab. Daher stieß der Kampf für die Entmachtung des deutschen Imperialismus nicht nur auf den erbitterten Widerstand des deutschen Monopolkapitals, sondern auch auf den der imperialistischen Siegermächte. Ungeachtet aller Gegensätze leisteten deshalb die imperialistischen Mächte dem deutschen Konkurrenten Hilfe bei der Niederschlagung der revolutionären deutschen Arbeiterklasse. Das gemeinsame Ziel war, Deutschland in eine Speerspitze gegen den ersten sozialistischen Staat zu verwandeln und einen internationalen Kreuzzug gegen die junge Sowjetmacht vorzubereiten.

Gegen die volksfeindliche Politik der in- und ausländischen Imperialisten wandte sich am entschiedensten die KPD. Sie wies darauf hin, daß die Herstellung eines guten Verhältnisses zu dem jungen Sowjetstaat eine Lebensfrage Deutschlands sei. Die Gründung der KPD - das bedeutsamste Ereignis der Novemberrevolution - stellte eine Wende in der Geschichte der deutschen Arbeiterbewegung und des deutschen Vol- 
kes dar. ${ }^{4}$ Erstmalig war nun auch in Deutschland eine revolutionäre marxistische Kampfpartei entstanden, die „die Fragen des Staates und der Revolution in der Epoche des Ubergangs vom Kapitalismus zum Sozialismus grundsätzlich richtig beantwortete und sich zur Diktatur des Proletariats bekannte".5 Die Gründung der KPD bedeutete den ideologischen und organisatorischen Bruch mit Revisionismus und Zentrismus. Auf den Lehren von Marx und Engels fußend, führte die KPD die revolutionäre Entwicklungslinie in der deutschen Arbeiterbewegung fort und legte den Grundstein für die Bildung einer marxistisch-leninistischen Partei. Damit wurde die entscheidende Voraussetzung geschaffen, die Lebensfrage des deutschen Volkes zu lösen. Ausgehend von der Erkenntnis, daß Deutschland nur als friedliebender, demokratischer Staat eine echte Perspektive hat, richtete die KPD den Hauptstoß gegen den deutschen Imperialismus und Militarismus und orientierte auf die revolutionäre Umgestaltung der gesellschaftlichen Verhältnisse zugunsten der Arbeiterklasse und ihrer Verbündeten. Ihre grundsätzliche Klarheit in der Machtfrage befähigte sie dazu, den Massen den Klassencharakter der Weimarer Republik zu enthüllen, die Koalitionspolitik der rechten sozialdemokratischen Führer aufzudecken und gegen die Illusionen über den bürgerlichen Parlamentarismus anzukämpfen.

Nach der Novemberrevolution galt es, die Lehre zu ziehen, daß es in dem hochindustrialisierten Deutschland mit einem starken staatsmonopolistischen Kapitalismus nicht möglich war, in einem Sprung die Diktatur des Proletariats zu erreichen. ${ }^{6}$ Vielmehr mußten die revolutionären Kräfte die Mehrheit der Arbeiterklasse und der übrigen Werktätigen um sich sammeln, um die in der Novemberrevolution errungenen demokratischen Rechte und Freiheiten zu verteidigen und die bisher nicht verwirklichten antiimperialistischen demokratischen Forderungen durchzusetzen. In solchen gemeinsamen antiimperialistischen Aktionen konnten die Volksmassen an den Kampf um die Macht herangeführt werden. Die Erfahrungen eines erbitterten Klassenkampfes sowie die ständige ideologische Auseinandersetzung mit Revisionismus und linkem Sektierertum förderten den Wachstumsprozeß der KPD. Im Ergeb-

4 Ulbricht, Walter, Fünfzig Jahre Kommunistische Partei Deutschlands. Ansprache auf der Festveranstaltung am 30. Dezember 1968 in Berlin anläßlich des 50. Jahrestages der Gründung der KPD, Berlin 1969, S. 5.

5 Geschichte der deutschen Arbeiterbewegung, a. a. O., S. 177.

6 Vgl. Ulbricht, Walter, Referat zum „Grundriß der Geschichte der deutschen Arbeiterbewegung“, in: Einheit, August 1962, Sonderheft, S. 33. 
nis dieser Entwicklung erarbeitete die Partei im Oktober 1919 in ihren Leitsätzen über kommunistische Grundsätze und Taktik ${ }^{7}$ die Grundlinie des Kampfes gegen den deutschen Imperialismus und Militarismus. Sie orientierte auf die gemeinsame Verteidigung der revolutionären Errungenschaften gegen die Angriffe des Klassengegners und wies damit der deutschen Arbeiterklasse und den anderen Werktätigen Richtung und Ziel. Die Abwehrkämpfe 1919, an deren Spitze KPD und linke USPD standen, schufen günstige Ausgangsbedingungen für das Zustandekommen der Aktionseinheit im Kapp-Putsch. In realer Einschätzung der volksfeindlichen Pläne der deutschen Imperialisten und Militaristen warnte die KPD eindringlich vor der Gefahr einer Militärdiktatur, weil diese nicht nur die deutsche Arbeiterklasse, sondern auch den Sieg des Sozialismus in Sowjetrußland bedrohte. Die KPD betonte deshalb immer wieder, daß die historische Mission der deutschen Arbeiterklasse nur durch das Bekenntnis zum proletarischen Internationalismus gelöst werden könne und die Verteidigung der sozialistischen Revolution in Sowjetrußland auch im Interesse des deutschen Volkes läge.

Die rechten sozialdemokratischen Führer hingegen verbreiteten mit ihrer Konzeption des „dritten Weges“ die Illusion, daß es möglich sei - ohne sozialistische Revolution -, vom Boden der kapitalistischen Gesellschaft aus mit Hilfe des Parlamentarismus und der bürgerlichen Demokratie den Sozialismus zu verwirklichen. Sie leugneten die Allgemeingültigkeit der Lehren der Oktoberrevolution und verleumdeten die revolutionären Kräfte in Deutschland und Sowjetrußland. Indem die Rechtsopportunisten die aus der nichtvollendeten Novemberrevolution hervorgegangene bürgerlich-parlamentarische Demokratie als die Verwirklichung der Sehnsucht der Volksmassen nach Demokratie und Sozialismus priesen, wollten sie die Massenforderungen abfangen und ein Gegenmodell zur sozialistischen Demokratie schaffen. Die Integrierung einer Anzahl von SPD-Funktionären in den bürgerlichen Staatsapparat von der Regierungsebene aus bis hinunter in die örtlichen Verwaltungsorgane begünstigte bei einem großen Teil der deutschen Werktätigen die von den rechten sozialdemokratischen Führern genährten bürgerlich-parlamentarischen Illusionen, daß es möglich sei, ohne

7 Vgl. die Materialien über den 2. Parteitag der KPD vom 20.-23. Oktober 1919 in Heidelberg und anderen Orten, in: Dokumente und Materialien zur Geschichte der deutschen Arbeiterbewegung, hg. v. Institut für Marxismus-Leninismus beim ZK der SED, Berlin 1966, Bd. VII, 1. Halbbd., S. 131 bis 140 . 
grundlegende Änderung der Machtverhältnisse an der Machtausübung beteiligt zu sein. Durch die revisionistischen Theorien über Staat und Revolution wurde die Mehrheit der Arbeiterklasse daran gehindert zu erkennen, daß die Voraussetzung für die Lösung der nationalen Lebensfrage des deutschen Volkes nur die politische und ökonomische Entmachtung des deutschen Imperialismus und Militarismus ist.

Die Erfahrungen aus den Kämpfen der Novemberrevolution reichten noch nicht aus, das Bewußtsein der Massen soweit zu entwickeln, daß sie sich vom Einfluß der Revisionisten und Opportunisten befreiten. Der Zustrom kleinbürgerlicher Elemente infolge ihrer ökonomischen Verelendung durch Krieg und Nachkrieg stärkte die Massenbasis der Sozialdemokratie und erleichterte die Verbreitung der antirevolutionären Konzeption der rechten Führer. Ihre Paktiererpolitik bedeutete eine Unterwerfung der Arbeiterklasse unter die Interessen des Monopolkapitals. Die gemeinsame ideologische Basis für Revisionisten und Imperialisten im Kampf gegen die Gesetzmäßigkeiten der neuen Epoche waren Antikommunismus und Nationalismus.

Im Ringen um einen kapitalistischen oder sozialistischen Weg in Deutschland kam der USPD, der in den Industriegebieten zahlenmäßig stärksten Partei, eine große Bedeutung zu. Die Haltung der rechten Führer der USPD ,wurde durch den Versuch einer Aussöhnung der zwei Klassenlinien, durch den Widerspruch zwischen ihrer bürgerlichen Klassenposition und der Rücksichtnahme auf die revolutionär-gesinnten Mitglieder und Anhänger der USPD bestimmt“. ${ }^{8}$ Die rechten Führer der USPD gaben sich als entschiedene Verfechter des Sozialismus und der Revolution aus, distanzierten sich mit Worten von der Koalitionspolitik der Revisionisten und behaupteten, für die Massenforderung nach revolutionärer Umgestaltung des imperialistischen Systems einzutreten. In der Praxis aber unterwarf sich die rechte USPD-Führung in allen entscheidenden Fragen der konterrevolutionären Politik der rechten sozialdemokratischen Führer.

Weil die Grundfrage unserer Epoche in der Novemberrevolution nicht gelöst werden konnte, wirkten die Gesetzmäßigkeiten, aus denen die Revolution erwachsen war, weiter. Zwar hatten die revolutionären Kräfte die antiimperialistische demokratische Revolution nicht zu Ende

8 Diehl, Ernst, Die Bedeutung der Novemberrevolution 1918, a. a. O., S. 22. 
führen können, aber es war der Konterrevolution auch nicht gelungen, die KPD zu vernichten. Die von der Großen Sozialistischen Oktoberrevolution ausgehenden Ideen des Sozialismus erfaßten über die Arbeiterklasse hinaus immer breitere Volksschichten und verstärkten deren $\mathrm{Haß}$ gegen das imperialistische System. Der unüberbrückbare Gegensatz zwischen den Interessen der Imperialisten und Militaristen einerseits und denen des Volkes andererseits hatte sich zunehmend verschärft.

In dem verzweifelten Bemühen, einen Ausweg aus der für sie prekären Situation zu finden, entwickelten sich zwei verschiedene Gruppierungen innerhalb der Monopolbourgeoisie, die zwar eine unterschiedliche Taktik $^{9}$ verfolgten, deren Strategie aber in jedem Fall auf eine Konsolidierung des Kräfteverhältnisses zugunsten des Imperialismus hinauslief. Während die „wendigeren“ Monopolisten den bürgerlichen Parlamentarismus zur Bindung der Massen an den Staat auszunutzen trachteten und deshalb auch bereit waren, die Zusammenarbeit mit den Opportunisten noch eine Zeitlang fortzusetzen, erblickten die reaktionärsten Kräfte des deutschen Imperialismus und Militarismus in einem sofortigen konterrevolutionären Staatsstreich die einzige Möglichkeit zur Überwindung ihrer labilen politischen Herrschaft.

Der von den extremsten Monopolisten, Junkern und Militaristen im Frühjahr 1920 inszenierte Kapp-Putsch sollte die im Ergebnis der Novemberrevolution entstandene bürgerlich-demokratische Republik beseitigen und eine Militärdiktatur errichten, um die uneingeschränkte Herrschaft des Finanzkapitals in Deutschland zu sichern und die revolutionären Kräfte endgültig niederzuschlagen. ${ }^{10}$ Darüber hinaus sollte der Putsch die innenpolitischen Voraussetzungen für neue Kriegsabenteuer schaffen und vornehmlich eine offene und aktive Beteiligung Deutschlands am Kampf gegen die junge Sowjetmacht vorbereiten. Mit der antisowjetischen Stoßrichtung dieses Staatsstreiches befanden sich die Verschwörer in Übereinstimmung mit den Plänen solcher enragierten Sowjetfeinde, wie des britischen Kriegsministers Winston Churchill, der einen neuen Kreuzzug des Weltimperialismus gegen den ersten siegreichen Arbeiter-und-Bauern-Staat der Geschichte vorbereitete.

9 Vgl. hierzu Ruge, Wolfgang, Zur Taktik der deutschen Monopolbourgeoisie im Frühjahr und Sommer 1919, in: ZfG, 6/1963, S. 1088-1117.

$10 \mathrm{Vgl}$. hierzu Geschichte der deutschen Arbeiterbewegung, a. a. O., S. 265 bis 266. 
Der Putsch der aggressivsten Vertreter des deutschen Imperialismus war nur möglich, weil die nicht entmachteten deutschen Imperialisten und Militaristen seit der Novemberrevolution erstarkt waren und die antikommunistische Politik der bürgerlichen Parteien und der rechten sozialdemokratischen Führung den Boden für einen solchen konterrevolutionären Vorstoß vorbereitet hatte.

Die von den Alliierten geforderte Reduzierung des deutschen Heeres auf 100000 Mann bis zum April 1920 veranlaßte die Putschisten, den Staatsstreich zu einem Zeitpunkt durchzuführen, wo ihr militärisches Kräftepotential dies noch zu erlauben schien. Die extremistischen Kreise um Kapp, Lüttwitz und Ludendorff glaubten, angesichts der Spaltung der Arbeiterklasse sicher zu sein, daß der Putsch gelingen würde. Darüber hinaus hofften sie, daß die Arbeiter nicht bereit wären, eine Republik zu verteidigen, die durch das Noskesystem diskreditiert war.

Die deutsche Arbeiterklasse beantwortete jedoch den Angriff der Putschisten auf die Lebensinteressen des deutschen Volkes mit der Herstellung der Aktionseinheit. Uberall in Deutschland bildeten sich Einheitsfrontorgane wie Streikleitungen, Aktionsausschüsse und Vollzugsräte aus Vertretern aller drei Arbeiterparteien und der Gewerkschaften, die ungeachtet unterschiedlicher politischer Konzeptionen gemeinsam den Kampf gegen die Militärdiktatur aufnahmen. Daß sich die Gewerkschaften, die größte Organisation der werktätigen Massen, in die Streikfront einreihten, war von besonderer Bedeutung. Erstmalig beteiligten sich auch die Landarbeiter in großem Umfang an einer solchen gemeinsamen proletarischen Aktion. Insgesamt nahmen über 12 Millionen Werktätige den Kampf gegen die Konterrevolution auf. In der vordersten Front standen wiederum die Kommunisten und die linken Kräfte der USPD.

Der konsequente Kampf der KPD gegen Imperialismus und Militarismus und ihre wiederholten Warnungen vor konterrevolutionären Putschen trugen wesentlich zur Herausbildung der Aktionseinheit bei. Die Bezirks- und Ortsorganisationen der KPD traten entschlossen in den Abwehrkampf ein. Die in den Einheitsfront- und Kampforganen der Arbeiter vertretenen Kommunisten waren die entschiedensten Verfechter konsequenter Maßnahmen gegen die Konterrevolution. Die in Berlin anwesenden Vertreter der Zentrale der KPD, die in ihrem Aufruf vom 13. März 1920 zwar die Notwendigkeit entschiedener Aktionen gegen die Militärdiktatur betonten, beschlossen unter dem Einfluß linker Führer der Berliner Bezirksleitung - gegen den Widerstand einer 
Minderheit in der Zentrale -, zu diesem Zeitpunkt noch nicht zum Generalstreik aufzufordern. Diese auf Unklarheiten über das dialektische Verhältnis des Ringens um Demokratie und Sozialismus be-ruhende Stellungnahme korrigierte die Zentrale bereits am nächsten Tage, indem sie zur gemeinsamen Bekämpfung der Putschisten aufrief. Mit ihrer Forderung nach Entwaffnung der Konterrevolution und Bewaffnung der Arbeiterklasse sowie durch die Aufdeckung des imperialistischen Klassencharakters des Putsches und der Rolle der Koalitionsregierung orientierte die KPD die Volksmassen auf das Ziel des antiimperialistischen Kampfes in der konkreten Situation. Der Abwehrkampf gegen die Putschisten mußte dazu ausgenutzt werden, die Konterrevolution insgesamt zu zerschlagen, die demokratischen Rechte und Freiheiten zu erweitern und die Positionen der Arbeiterklasse zu stärken. Deshalb beschränkten sich die Arbeiter unter Führung der KPD und linker USPD-Mitglieder nicht nur auf den Generalstreik, sondern gingen zum Kampf mit der Waffe in der Hand über. Vornehmlich in den Industriegebieten kam es zu bewaffneten Kämpfen der Arbeiterwehren gegen die konterrevolutionären Militärverbände. Im Ruhrgebiet erreichten die bewaffneten Auseinandersetzungen ihren Höhepunkt und führten $\mathrm{zu}$ einer zeitweiligen Befreiung dieses wichtigen Industriereviers von den konterrevolutionären Truppen.

In welchem Ausmaß die Kampfentschlossenheit der antiimperialistischen Kräfte gegen die Militärdiktatur die Haltung der herrschenden Kreise, die den Volkskampf fürchteten, beeinflußte, wird daraus ersichtlich, daß die politisch wendigeren Teile der Großbourgeoisie sich abwartend verhielten, die mit den Putschisten sympathisierende Monopolgruppe unter Führung des mit Kapp in enger Verbindung stehenden Ruhrindustriellen Hugo Stinnes es nicht wagte, sich offen zu dem Staatsstreich zu bekennen. Viele hohe Militärs sahen sich angesichts der für sie unerwarteten Wendung der Dinge zu der Taktik gezwungen, lediglich indirekt - unter der Parole „Aufrechterhaltung der Ruhe und Ordnung" - der Putschregierung Vorschub zu leisten.

Der einmütige Generalstreik und die Erfolge der Arbeiter im bewaffneten Kampf führten zur Niederschlagung des Putsches und verhinderten die Errichtung der Militärdiktatur. Die Tatsache, daß unter dem Eindruck der Kampfbereitschaft des Proletariats auch breite Teile des demokratischen Bürgertums mitgerissen wurden, zeigt, daß die Einbeziehung der Mittelschichten in die antiimperialistische demokratische Front realisierbar ist. Die Aktion gegen Kapp-Lüttwitz stellte die größte 
Massenbewegung seit der Novemberrevolution und die wirkungsvollste Einheitsaktion in der Weimarer Republik dar. ${ }^{11}$

Die Niederschlagung der Militärdiktatur in Deutschland war zugleich von internationaler Bedeutung, denn die deutschen Arbeiter trugen dazu bei, „den Plan der Weltreaktion zu vereiteln, Deutschland aktiv in den antisowjetischen Kampf einzubeziehen“.12

Der Sieg der Arbeiterklasse über die Putschisten beweist, daß es möglich ist, die Imperialisten und Militaristen zu schlagen. Als unmittelbares Ergebnis des Volkskampfes veränderte sich zeitweilig das Kräfteverhältnis in Deutschland. Die Aktionseinheit der Arbeiterklasse eröffnete die Möglichkeit, eine Wende in der Politik herbeizuführen und wahrhaft demokratische Verhältnisse zu schaffen. Es galt, den Imperialismus und Militarismus zurückzudrängen, mit der Koalitionspolitik der rechten sozialdemokratischen Führer zu brechen und eine Arbeiterregierung zu bilden. Deren Aufgabe mußte es sein, die demokratischen Rechte und Freiheiten zu erweitern und günstigere Ausgangsbedingungen für den Kampf der revolutionären Kräfte zu schaffen. Die Bildung einer solchen Arbeiterregierung hätte sowohl im Interesse der deutschen Arbeiterklasse gelegen als auch die demokratische Entwicklung des Volkes vorangetrieben. Wie sich aus der in vielen Teilen Deutschlands erhobenen Forderung nach einer Arbeiterregierung erwies, entsprach diese dem Streben breiter Teile der Werktätigen und war dem Reifegrad der deutschen Arbeiterklasse angemessen. ${ }^{13}$ Während die KPD die Massen darauf orientierte, die Einheitsfront des Proletariats zu erhalten und die errungenen Erfolge auszubauen, strebten die konterrevolutionären Kräfte danach, diese Aktionseinheit, die sie am stärksten fürchteten, zu zerschlagen. Sie bedienten sich dabei der rechten sozialdemokratischen Führer. Diese suchten zunächst mit Hilfe von Kompromissen, wie dem Neunpunkteprogramm und dem Bielefelder Abkommen, Teile des Proletariats zur Einstellung des Kampfes zu bewegen und die revolutionären Kräfte zu isolieren. Ihr Ziel war, mittels des Antikommunismus die Einheitsfront der Arbeiterklasse auseinanderzumanövrieren und

11 Vgl. Matern, Hermann, Aktionseinheit schlug die Reaktion. Zum 40. Jahrestag der Niederschlagung des Kapp-Putsches durch die Aktionseinheit der Arbeiterklasse und aller demokratischen Kräfte, in: Einheit, 3/1960, S. $442-456$.

12 Vgl. Geschichte der deutschen Arbeiterbewegung, a. a. O., S. 275.

$13 \mathrm{Vgl}$. dazu Ulbricht, Walter, Referat zum „Grundriß der Geschichte der deutschen Arbeiterbewegung", a. a. O., S. 35. 
die bisherige Koalitionspolitik fortzusetzen. Während die rechten Führer der SPD und der USPD die Aktionseinheit der Arbeiterklasse sprengten, ging die Konterrevolution zum Gegenangriff über. In blutigen Terrorfeldzügen suchte sie die von den Werktätigen in der Abwehr des Kapp-Putsches errungenen Machtpositionen zu zerschlagen.

Der Volkskampf gegen die Errichtung einer offenen Militärdiktatur läßt die Grundfragen des Kampfes zwischen Kapitalismus und Sozialismus offen zutage treten. Die bei der Niederschlagung des Kapp-Putsches gewonnenen Erfahrungen bilden eine Quelle wichtiger Lehren für die Arbeiterklasse und ihre Verbündeten in dem gegenwärtigen Kampf gegen den staatsmonopolistischen Kapitalismus.

Am Beispiel des Kapp-Putsches offenbart sich, daß der Imperialismus eine tödliche Gefahr für das gesamte Volk darstellt, daß imperialistische Herrschaft und Interessen der Volksmassen unvereinbar sind und die stärkste Waffe der Arbeiterklasse im Kampf gegen den Imperialismus ihre Aktionseinheit ist. Die Aktionsgemeinschaft gegen die Kapp-Putschisten vermittelt die historische Lehre, daß es möglich ist, trotz Meinungsverschiedenheiten in prinzipiellen Fragen, zu gemeinsamen Kampfaktionen gegen Imperialismus und Militarismus zu kommen.

Bei der Errichtung des ersten Arbeiter-und-Bauern-Staates in der deutschen Geschichte wurden die Lehren und Schlußfolgerungen aus dem jahrzehntelangen Kampf der deutschen Arbeiterklasse, in dem die Niederschlagung des Kapp-Putsches ein bedeutsames Ereignis darstellt, berücksichtigt. Die Ideologen des spätkapitalistischen Systems in Westdeutschland sehen sich daher veranlaßt, die Erfahrungen der deutschen Arbeiterklasse im Kampf gegen Imperialismus und Militarismus zu verfälschen. In der gegenwärtigen Situation, in der die Ideologie zu einem Hauptfeld der internationalen Klassenauseinandersetzung wird, tritt die Doppelfunktion der imperialistischen Historiographie besonders deutlich in Erscheinung: Einerseits übernimmt sie die weltanschauliche Beeinflussung der Volksmassen und andererseits beteiligt sie sich aktiv an der Konzipierung der imperialistischen Strategie und Taktik. Ihre Aufgabe ist es, politische Leitbilder aus der Geschichte heraus zu schaffen und Modelle $\mathrm{zu}$ entwickeln, die geeignet sind, die Arbeiterklasse in die spätkapitalistische Gesellschaft $\mathrm{zu}$ integrieren und sie davon abzuhalten, die Macht in ihre eigenen Hände zu nehmen.

Die imperialistischen Historiker und Publizisten können jedoch nicht mehr die Ergebnisse der marxistisch-leninistischen Geschichtsschreibung 
über den von der Arbeiterklasse geführten Volkskampf gegen Kapp und Lüttwitz ignorieren. So haben sie die bisherigen Thesen bürgerlicher Historiker, daß der Kapp-Putsch am Beamtenstreik, an der Passivität der Monopolisten oder an der Kreditverweigerung der Reichsbank für die Putschisten gescheitert sei, fallengelassen und sehen sich gezwungen, einzugestehen, daß die Arbeiter im Frühjahr 1920 die demokratischen Rechte verteidigten und die Putschisten schlugen. Diese Tatsache wird indes verfälscht, indem man sowohl Inhalt und Methoden dieses Volkskampfes als auch seine Zielstellung und Ergebnisse im imperialistischen Sinne manipuliert. Den einmütigen Generalstreik der Arbeiterklasse in ganz Deutschland, der neben den bewaffneten Kämpfen die Hauptürsache für die Niederlage des Putsches war, stellt man lediglich als eine für die damalige konkrete Situation geeignete Maßnahme hin. Gleichzeitig warnen die bürgerlichen Historiker die Gewerkschaften vor der „zweischneidigen Waffe“ des Generalstreiks. Die herrschenden Kreise in Westdeutschland wissen genau, daß Massenaktionen mit Beteiligung der Gewerkschaften auch in der Gegenwart in der Lage wären, die aggressiven Pläne der abenteuerlichen Monopolisten zu vereiteln.

Im Zeichen der als Gegengewicht gegen den Marxismus-Leninismus entwickelten Integrationskonzeption ${ }^{14}$ interpretieren die Ideologen des staatsmonopolistischen Systems, gestützt auf Argumente rechter Sozialdemokraten ${ }^{15}$, den Kampf der Arbeiterklasse gegen die Kapp-Putschisten lediglich als Kampf für die Erhaltung der Weimarer Republik. Damit werden die bereits damals von den rechten sozialdemokratischen Führern ausgegebenen Parolen neu belebt, mit deren Hilfe es nach dem Sturz der Putschregierung gelang, den Volkskampf abzuwürgen und die Koalition mit dem Monopolkapital fortzusetzen. Die Reduzierung der Klassenkampfziele auf Erhaltung der bürgerlichen Republik und der bürgerlichen Demokratie läuft darauf hinaus, den antiimperialistischen Charakter des Volkskampfes zu verschleiern und die Arbeiterklasse zu einem in die klassenneutrale „demokratische Bewegung“ schlechthin integrierten Bestandteil der formierten bürgerlichen Gesell-

14 Siehe hierzu Lozek, Gerhard/Schmidt, Walter, Die „Integrations“konzeption - Grundlage bürgerlicher Verfälschung der Geschichte der Arbeiterbewegung, in: Einheit, 10/1968, S. 1268-1279.

15 Vgl. hierzu u. a. die anläßlich des 50. Jahrestages der Novemberrevolution erschienenen Artikel führender rechter Sozialdemokraten in der Zeitschrift der SPD „Die Neue Gesellschaft“, 6/1968. 
schaft zu manipulieren, um ihre führende Rolle im Kampf gegen die Konterrevolution bestreiten zu können.

Entsprechend der berüchtigten Totalitarismusdoktrin diffamieren imperialistische und rechte sozialdemokratische Historiker und Publizisten die revolutionären Kräfte der deutschen Arbeiterklasse mit der KPD an der Spitze als „antidemokratisch“ und werten deren Forderungen nach Herstellung wahrhaft demokratischer Verhältnisse als "Störung" des Integrationsprozesses der Arbeiterklasse in die bürgerliche Klassengesellschaft. Die Bemühungen der KPD zur Aufrechterhaltung der Aktionseinheit der Arbeiterklasse bezeichnen diese Ideologen lediglich als "taktisches Manöver".

Der Hauptstoß der imperialistischen Ideologen richtet sich jedoch gegen die Aneignung des Leninismus durch die KPD, weil sie erkannt haben, daß die Arbeiterklasse nur mit Hilfe der marxistisch-leninistischen Strategie und Taktik den Imperialismus zu stürzen vermag. Mit der systematischen Abwertung bzw. weitgehenden Verschweigung der Aktionseinheit der Arbeiterklasse verfolgt die bürgerliche Historiographie das Ziel, das Zustandekommen gemeinsamer Aktionen der antiimperialistischen Kräfte in der Gegenwart zu vereiteln.

In der jüngst erschienenen Spezialstudie des Conze-Schülers Erger über den Kapp-Putsch wird beispielsweise der Reichswehr das Verdienst zugeschrieben, den Putsch beendet zu haben. Auf diese Art soll nicht nur der Militarismus in der Weimarer Republik rehabilitiert, sondern auch die Bundeswehr heute als ein „Faktor der Stabilität der bürgerlichen Ordnung" in Westdeutschland hingestellt werden. Um davon abzulenken, daß der Militarismus stets das Werkzeug des Imperialismus war und ist und entgegen der Tatsache, daß die einig handelnde Arbeiterklasse die Errichtung einer Militärdiktatur verhinderte, behauptet Erger, „daß der Putsch von keiner Seite niedergeschlagen worden“ sei, sondern „durch den Dualismus von Kapp und Lüttwitz von vornherein zum Scheitern verurteilt" war. $^{16}$

Nach der Niederschlagung des Kapp-Putsches begannen die revolutionären Kräfte der deutschen Arbeiterbewegung, grundlegende Schlußfolgerungen für ihre Strategie und Taktik zu ziehen. Die Erkenntnis über die Bedeutung der Aktionseinheit trieb den ideologischen Reifeprozeß der Arbeitermassen voran und stärkte die Idee des proletari-

16 Erger, Johannes, Der Kapp-Lüttwitz-Putsch. Ein Beitrag zur deutschen Innenpolitik 1919/20, Düsseldorf 1967, S. 300. 
schen Internationalismus, wie sich in der Aktion „Hände weg von Sowjetrußland!“ im Sommer 1920 zeigte. Die gemeinsamen Klassenkampferfahrungen gegen die Putschisten beschleunigten den Klärungsprozeß vornehmlich unter den linken Mitgliedermassen der USPD, die sich Ende 1920 mit der KPD zur VKPD vereinigten. Damit war die $\mathrm{KPD}$ in historisch kurzer Zeit „als erste kommunistische Partei in einem hochindustrialisierten Lande $\mathrm{zu}$ einer Massenpartei geworden “. ${ }^{17}$

Mit Unterstützung der Kommunistischen Internationale und Lenins, der hervorgehoben hatte, daß der Klassenkampf nur dann erfolgreich sein kann, „wenn die revolutionäre Vorhut des Proletariats dessen überwältigende Mehrheit hinter sich hat" ${ }^{18}$, arbeitete die KPD eine Strategie und Taktik aus, die es ermöglichte, die Volksmassen unter den Bedingungen eines hochentwickelten kapitalistischen Staates an die Eroberung der Macht heranzuführen. In schöpferischer Anwendung der marxistisch-leninistischen Staatstheorie galt es, den Kampf für demokratische Verhältnisse weiterzuentwickeln, um schließlich die Grundfrage der Epoche zu lösen. Mit dem „Offenen Brief“ vom Januar 1921 an die Leitungen der Gewerkschaften, der SPD, USPD und KAPD beschritt die KPD einen Weg zur Herstellung einer proletarischen Einheitsfront, der von der Kommunistischen Internationale als beispielhaft eingeschätzt wurde. ${ }^{19}$ Die Verbindung des revolutionären Kampfes um den Sozialismus mit dem Ringen um die unmittelbaren Tagesforderungen der Werktätigen war geeignet, die Massen in den politischen Klassenkampf einzubeziehen.

Große Bedeutung für die Durchführung einer erfolgreichen Massenpolitik gewann die Losung von der Arbeiterregierung. Sie stellte eine Bereicherung der marxistisch-leninistischen Strategie und Taktik dar. Durch Gewinnung der Mehrheit der Arbeiterklasse und ihrer Verbündeten mußte eine Arbeiter- bzw. Gewerkschaftsregierung erzwungen werden, um die Herrschaft der deutschen Imperialisten und Militaristen zurückzudrängen und wahrhaft demokratische Verhältnisse zu schaffen. ${ }^{20}$

17 Vgl. Geschichte der deutschen Arbeiterbewegung, a. a. O., S. 310.

18 Lenin, W. I., Werke, Bd. 31, Berlin 1959, S. 179.

19 Vgl. Ulbricht, Walter, Die Kommunistische Internationale im Kampf gegen imperialistischen Krieg und Faschismus. Rede auf der wissenschaftlichen Tagung „50. Jahrestag der Kommunistischen Internationale“, in: Neues Deutschland v. 27. März 1969.

20 Vgl. derselbe, Fünfzig Jahre Kommunistische Partei Deutschlands, a. a. O., S. 16-17. 
Die prinzipielle Klärung der Einheitsfrontpolitik und der Bündnispolitik befähigte die KPD, im Kampf gegen den Hitlerfaschismus die Strategie und Taktik für die Schaffung einer demokratischen deutschen Republik auszuarbeiten. ${ }^{21}$ Mit Unterstützung der Kommunistischen Internationale entwickelte die KPD auf den Konferenzen in Brüssel und Bern die antifaschistische Volksfrontpolitik auf der Grundlage der Aktionseinheit der Arbeiterparteien und der Gewerkschaften.

Nach der gesetzmäßigen Niederlage des Imperialismus im zweiten Weltkrieg machte die KPD entsprechend ihrem Programm vom Juni 1945 die Volksfrontpolitik zum Ausgangspunkt für die demokratische Umgestaltung Deutschlands. Die Beseitigung der Machtgrundlagen des deutschen Imperialismus und Militarismus ermöglichte die Lösung der Grundfragen unserer Epoche. Durch die Herstellung der Aktionseinheit der Arbeiterklasse sowie durch die Einbeziehung aller antifaschistischen demokratischen Kräfte konnte im Osten Deutschlands der demokratische Neuaufbau eingeleitet werden. Unter Führung der SED, einer Partei neuen Typus, die sich von der marxistisch-leninistischen Strategie und Taktik leiten ließ, wurden in der DDR im schärfsten Klassenkampf, aber auf unblutige Weise zwei Revolutionen siegreich durchgeführt.

Die Deutsche Demokratische Republik ist das gesetzmäßige Ergebnis des jahrzehntelangen Kampfes der deutschen Arbeiterklasse um die Bewältigung der Grundfragen unserer Epoche. ${ }^{22}$ Ihre Gründung stellt den entscheidenden Wendepunkt der deutschen Geschichte dar. Sie schuf ein Beispiel für hochindustrialisierte Länder beim Ubergang vom Kapitalismus zum Sozialismus. Als erster sozialistischer Staat auf deutschem Boden verkörpert sie ,in Ubereinstimmung mit dem Entwicklungsgesetz unserer Epoche die nationale Alternative zum spätkapitalistischen System in Westdeutschland“.23

21 Vgl. hierzu Ittershagen, Siegfried/Karl, Heinz/Wimmer, Walter, Zur Entwicklung der Strategie und Taktik der KPD von der Novemberrevolution bis zur Brüsseler Konferenz (I. und II.), in: BzG, 1/1963, S. 32-52; 2/1963, S. 228-246.

2220 Jahre Deutsche Demokratische Republik (Thesen), in: Neues Deutschland v. 16. Januar 1969.

23 Vgl. die Rede Walter Ulbrichts in Moskau, in: Internationale Beratung der kommunistischen und Arbeiterparteien Moskau 1969, a. a. O., S. 281. 
Die vorliegende Quellenpublikation ${ }^{24}$ soll dokumentieren, wie es im antiimperialistischen Volkskampf unter Führung der deutschen Arbeiterklasse gelang, den Versuch der extremsten Konterrevolution zur gewaltsamen Beseitigung der Errungenschaften der Novemberrevolution und zur Errichtung einer Militärdiktatur zu zerschlagen. Im Mittelpunkt stehen solche Materialien, die Entwicklung, Verlauf und Ergebnisse des Volkskampfes erhellen. Besonderer Wert wurde darauf gelegt, die Herstellung der Aktionseinheit der Arbeiterklasse, die die Voraussetzung für die Niederschlagung des Putsches durch die demokratischen Kräfte bildete, mit Quellen zu belegen. Demzufolge enthält die Publikation vorwiegend Dokumente der Arbeiterparteien und Gewerkschaften sowie der Einheitsorgane der Arbeiterklasse. Aufrufe, Flugblätter, Presseartikel und sonstige Materialien von Aktionsausschüssen, Vollzugsräten und Arbeiterorganisationen geben Aufschluß sowohl über die gemeinsame Zielstellung des Kampfes als auch über das Weiterwirken bestehender unterschiedlicher Konzeptionen der einzelnen Abteilungen der Arbeiterbewegung. So ist es möglich, den Grad der Wirksamkeit der proletarischen Aktionen sichtbar werden zu lassen wie auch die Hemmnisse innerhalb des gemeinsamen Kampfes aufzudecken.

Um die Dialektik des Klassenkampfes zu demonstrieren, mußte auch die Politik der herrschenden Klassen quellenmäßig belegt werden. Neben den Materialien von seiten der Putschisten zur Vorbereitung und Durchführung des Staatsstreiches fanden solche Dokumente Aufnahme, aus denen die unterschiedlichen taktischen Konzeptionen der Monopolbourgeoisie sowie die Stellungnahme der bürgerlichen Parteien zum Putsch und zu den Massenkämpfen hervorgehen und weiterhin Quellen, die die Rolle des imperialistischen Staatsapparates auf seinen verschiedenen Ebenen beleuchten.

Die einzelnen Abschnitte der Publikation, die Auskunft über die Spezifik der Klassenauseinandersetzungen im regionalen Maßstab geben, sind als eine dialektische Einheit zu betrachten, die in ihrer Vielfalt den antiimperialistischen Volkskampf bei der Niederschlagung des KappPutsches insgesamt widerspiegelt.

An dieser Stelle möchte ich allen an der Fertigstellung dieser Quellenpublikation Beteiligten danken. Mein Dank gilt den Bearbeitern Herrn Dr. Erwin Könnemann (Leiter der Arbeitsgruppe), Frau Dr. Brigitte

2481 der hier publizierten Dokumente wurden dem Verlag Marxistische Blätter GmbH in Frankfurt am Main von uns zum Vorabdruck zur Verfügung gestellt. 
Berthold und Herrn Gerhard Schulze, die in mehrjähriger Arbeit in zentralen, regionalen und kommunalen Archiven, Bibliotheken und Museen das Material sammelten.

Es ist mir ein Bedürfnis, den Direktoren und den Mitarbeitern dieser Institutionen sowie den Kommissionen zur Erforschung der Geschichte der örtlichen Arbeiterbewegung bei den Bezirks- und Kreisleitungen der SED den Dank auszusprechen.

Wesentliche Unterstützung bei der konzeptionellen Gestaltung der Publikation gaben uns der stellvertretende Direktor des Instituts für Marxismus-Leninismus beim ZK der SED ${ }^{25}$, Herr Prof. Dr. Ernst Diehl, und der Sektorleiter, Herr Dr. Hans-Joachim Krusch. Ferner danke ich Herrn Dr. Joachim Böhm und Frau Dr. Evemarie Peters für die Einsatzbereitschaft bei der Fertigstellung der Publikation sowie für die umfangreichen Schreibarbeiten Frau Else Wünsche und Frau Melitta Schrank.

Halle (Saale), im November 1969

\section{Leo Stern}

25 Vom Institut für Marxismus-Leninismus beim ZK der SED wird eine Monographie von Könnemann, Erwin/Krusch, Hans-Joachim über die Niederschlagung des Putsches vorbereitet, in der die im Vorwort skizzierte Problematik ausführlich behandelt wird. 
